\title{
Iron-regulated gene ireA in avian pathogenic Escherichia coli participates in adhesion and stress-resistance
}

\author{
Yaxin Li, Jianjun Dai, Xiangkai Zhuge, Haojin Wang, Lin Hu, Jianluan Ren, Ling Chen, Dezhi Li and Fang Tang*
}

\begin{abstract}
Background: Avian pathogenic Escherichia coli (APEC) causes avian colibacillosis, which results in economic and welfare costs in the poultry industry worldwide. The pathogenesis of avian pathogenic E. coli strains is not well defined. Here, the function of an outer membrane protein encoded by the ireA gene of avian pathogenic $E$. coli strain DE205B was investigated.

Results: The ireA gene was distributed in $32.9 \%(46 / 140)$ of tested E. coli strains, with high percentages in the phylogenetic ECOR groups B2 $(58.8 \%, 10 / 17)$ and D $(55.9 \%, 19 / 34)$. The gene expression level of ireA of APEC strain DE205B in high Fe M9 media was 1.8 times higher $(P<0.05)$ than that in low Fe M9 media. An ireA deletion mutant and complementary strain were constructed. Compared with the wild-type strain DE205B, the expression of most ferric uptake genes in the ireA deletion mutant were significantly upregulated $(P<0.05)$. The adhesion ability of the ireA deletion mutant to DF-1 cells was significantly decreased. The survival rate of ireA deletion mutant was reduced $21.17 \%(P<0.01), 25.42(P<0.05)$ and $70.0 \%(P<0.01)$ under alkali, high osmolarity, and low temperature $\left(4^{\circ} \mathrm{C}\right)$ conditions, respectively, compared with the wild-type strain.
\end{abstract}

Conclusions: The results suggested that the protein encoded by the iron-regulated gene ireA has roles in adhesion and stress resistance in avian pathogenic E. coli.

Keywords: APEC, IreA, Virulence, Adhesion, Stress resistance

\section{Background}

Avian pathogenic Escherichia coli (APEC), a subgroup of extra-intestinal pathogenic E. coli (ExPEC) causes avian colibacillosis and imposes economic losses on the poultry industry worldwide [1]. However, the pathogenesis of APEC is poorly understood. Many virulence genes have been studied to identify virulence factors in APEC, including those involved in adhesion, iron-regulation, toxin/cytotoxin production and serum resistance [2]. Iron is an essential element involved in important biological processes [3]. Biological activities in cells, such as peroxide reduction, nucleotide biosynthesis and electron transport, are facilitated by iron ions [4]. Extra-intestinal sites have low iron contents; therefore, ExPEC strains struggle to take up iron from

\footnotetext{
* Correspondence: tangfang@njau.edu.cn

Key Laboratory Animal Bacteriology, Ministry of Agriculture, College of veterinary medicine, Nanjing Agricultural University, No.1 Weigang, Nanjing 210095, Jiangsu province, People's Republic of China
}

the host during infection [5]. During natural infection, the initiation, progression and transmission of most bacterial infections depend on the ability of the invading pathogen to acquire iron from the complicated environment [6]. During iron acquisition, the cell must produce transmembrane receptors for siderophores that chelate iron ions [7]. There are various receptors that chelate iron ions encoded by bacterial genes, such as chuA, the SitABCD system, iron, iha, iutA, and ireA. Outer membrane protein ChuA participates in heme acquisition in enterohemorrhagic E. coli and uropathogenic E. coli (UPEC) strains, and is important for the pathogenicity of APEC [8, 9]. The SitABCD system, identified in the APEC strain MT512 by comparative genomic analysis, was reported to be associated with the pathogenicity of APEC $[9,10]$.

IreA was suggested to be involved in Fe acquisition and to act as an iron-regulated virulence gene in the blood- or urine-derived ExPEC E. coli isolated from 
humans [11]; however, its exact role in APEC strains remains unknown. Herein, an ireA deletion mutant was constructed to study the ireA gene function in the APEC strain DE205B.

\section{Results}

Prevalence of the ireA gene among $E$. coli Strains

As shown in Table 1, ireA was present in $32.9 \%(46 / 140)$ of $E$. coli strains, with $19.0 \%(12 / 63)$ in phylogenetic ECOR group A, $19.2 \%(5 / 26)$ in B1, $58.8 \%(10 / 17)$ in B2 and $55.9 \%(19 / 34)$ in group D (Additional file 1: Table $\mathrm{S} 1)$. Thus, the $\operatorname{ire} A$ gene was significantly more frequently distributed in the B2 and D groups than in the A and B1 groups.

\section{Expression of the ireA gene}

The ire $A$ gene expression was tested by Immunoblotting. Western blotting was performed with anti-His serum, showing expected fusion protein bands for ireA $(39 \mathrm{kDa})$ from strains DE205B. However, only fusion his protein $(18 \mathrm{kDa})$ was detected from the blank plasmid control (Fig. 1). These results indicated that ire $A$ was expressed under laboratory conditions.

\section{Gene expression of ireA in M9 media with different iron content}

The relative gene expression of ireA in low $\mathrm{Fe}$ M9 media was 1.8 times higher than that in high Fe M9 media $(P<0.01)$ (Fig. 2$)$.

\section{Growth curve}

The CFU of the wild-type strain DE205B, mutant strain DE205B $\triangle$ ireA and the complementary strain DE205BCAireA was monitored for $12 \mathrm{~h}$. There was no significant difference between the growth curves of the wild-type and mutant strains (Fig. 3), which indicated that deletion of ireA had no effects on the growth of DE205B.

\section{Expression variations of Ferric uptake system and adherence genes}

The expressions of $f e p C, f e o B$, chuT, fyuA, irp1, irp2, chuA and fepA were detected in the mutant and compared with their expressions in the wild-type strain DE205B. Most of the ferric uptake genes in the mutant

Table 1 Distribution of the ireA gene in Escherichia coli strains

\begin{tabular}{llll}
\hline ECOR group & Strain counts & Strain counts positive for ireA & Percentage \\
\hline A & 63 & 12 & $19.0 \%$ \\
B1 & 26 & 5 & $19.2 \%$ \\
B2 & 17 & 10 & $58.8 \%$ \\
D & 34 & 19 & $55.9 \%$ \\
Total & 140 & 46 & $32.9 \%$ \\
\hline
\end{tabular}

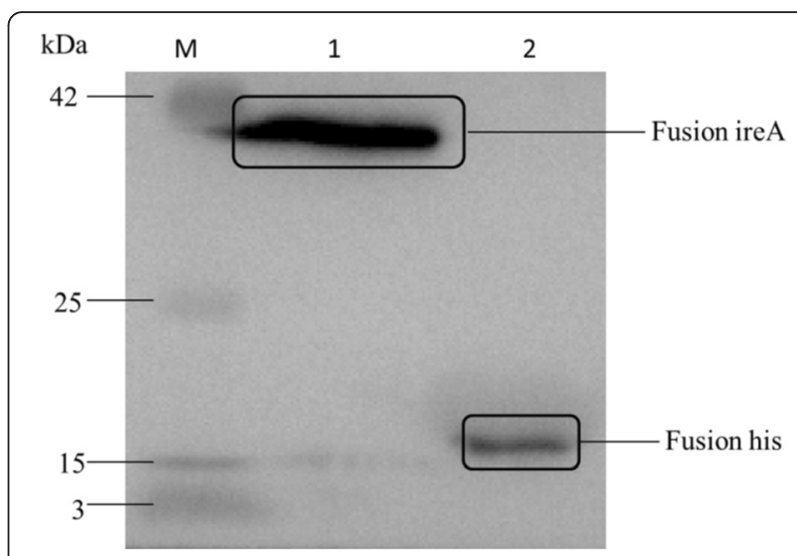

Fig. 1 Expression of ireA by western blotting. Expression of fusion ireA protein was detected by immunoblotting. Fusion ireA was detected in pET32a(+), while there is only his protein was detected in the blank plasmid. Lane $\mathrm{M}$, protein marker; lane 1, fusion ireA; lane 2, fusion his

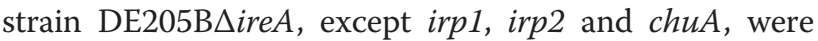
significantly upregulated $(P<0.05)$ (Fig. 4). Irp1, irp2 and $c h u A$ were non-significantly upregulated. Furthermore, the expressions of the eight ferric uptake genes in the complementary strain DE205BCAireA were restored to wild-type levels.

The expression of adherence genes $y f c O, y f c Q$, aufG, fmlD, fmlE, yadN and fimH showed no significant difference between the wild-type strain DE205B and the ireA mutant strain (Fig. 5).

\section{Adherence assays}

The number of mutant DE205B $\triangle$ ireA bacteria adhering to DF-1 cells was significantly decreased compared with

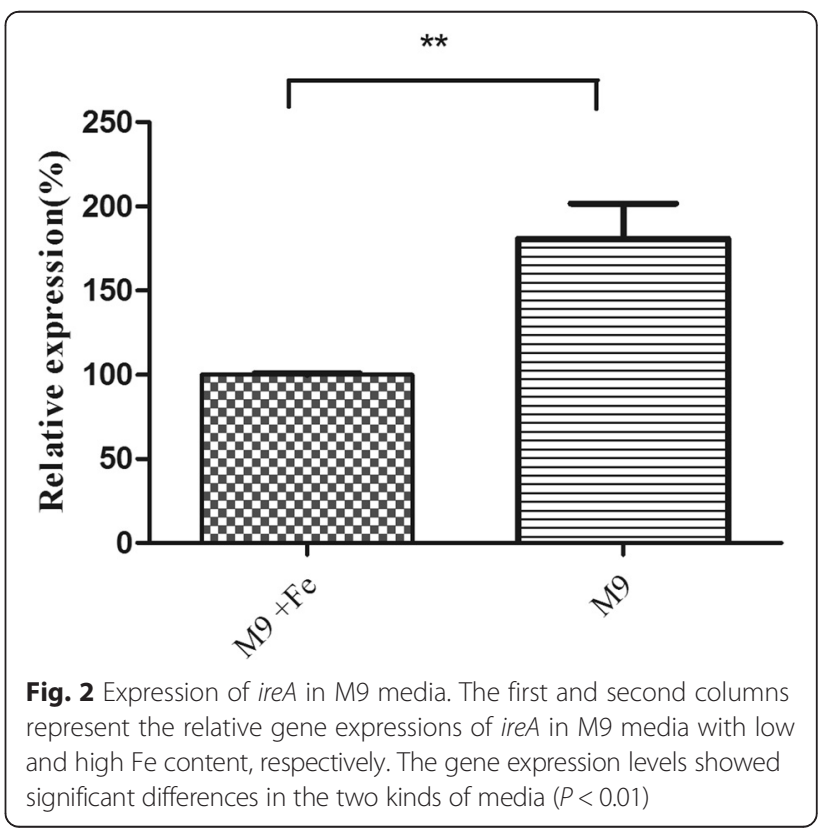




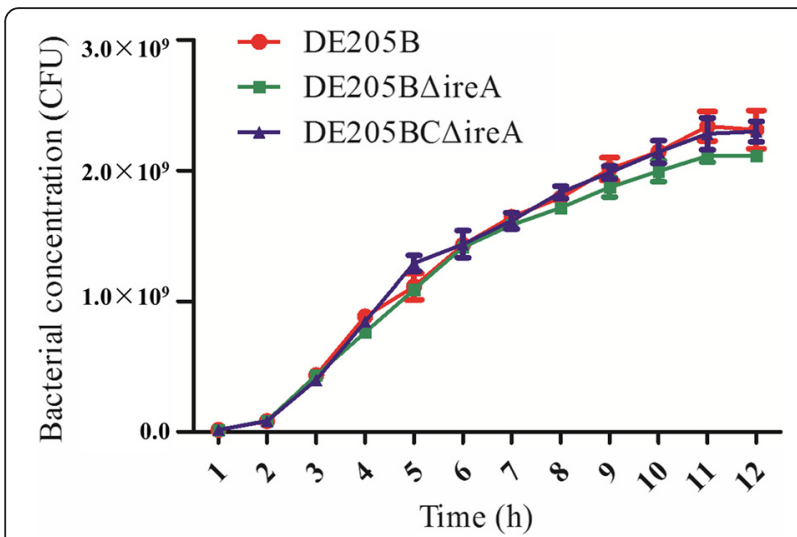

Fig. 3 Growth curve of different strains. Growth curves of the wild-type strain DE205B, mutant strain DE205BAireA and complementary strain DE205BC $\Delta$ ireA. Bacterial growth was estimated by plate counting as Colony Forming Units (CFU)

the number of DE205B wild-type adherent cells $(P<$ $0.05)$ (Fig. 6). The adhesion to DF-1 cells of complementary strain DE205BCAireA was partly restored to wildtype levels.

\section{Animal infection}

The $\mathrm{LD}_{50}$ of the wild-type, deletion mutant and complementary strains were $1.74 \times 10^{5}, 2.45 \times 10^{5}$, and $3.16 \times 10^{5}$, respectively (Table 2 ). The results showed that there was no significant difference between the $\mathrm{LD}_{50}$ of the wildtype and deletion mutant strains, indicating that the deletion of ireA gene has no obvious effect on the virulence of DE205B toward ducks.

\section{Determination of resistance to environmental stress}

Compared with the wild-type strain, the survival rate of the ireA deletion mutant was reduced by $21.17 \%(P<0.01)$ and
$25.42 \%(P<0.05)$ under alkali and high osmolarity conditions, respectively (Fig. 7). The resistance to alkali and high osmolarity conditions of the complementary strain DE205BCAireA was almost restored to wild-type levels. For the temperature challenge, compared to the wild type strain, the ireA deletion mutant was reduced $70.0 \%(P<0.01)$ under the low $\left(4{ }^{\circ} \mathrm{C}\right)$ temperature (Fig. 8). The resistance to low temperature of the complementary strain DE205BCAireAwas partly restored. The results showed that presence of the ire $A$ gene increased the stress-resistance of APEC strain DE205B.

\section{Discussion}

The ire $A$ gene is an iron-regulated gene and is involved in iron acquisition in human pathogenic E. coli isolates and our study proved this protein functions in APEC. Additionally, we identified two new functions of ireA using the deletion mutant. In the present study, ire $A$ was demonstrated to contribute to the adhesion to DF-1 cells. Moreover, the expressions of several adhesion genes were tested and the results showed no significant differences between wild-type and mutant strain, indicating that the ireA gene indeed plays a role in adhesion. Tarr et al., identified adhesin Iha from an O157:H7 strain of E. coli [12]. This adhesin shared high similarities with several identified or putative siderophore receptors. Siderophore receptor IrgA was reported to contribute to growth in the rabbit ileal loop model in vivo and to enhance virulence in an infant mouse model, suggesting a possible role in colonization [13, 14]. Here, we proved that iron-regulated gene ire $A$ plays a role in the adhesion of APEC strains. The ireA gene also increased stress-resistance under alkali and high osmolarity conditions, as well as underlow temperature. Thus, the

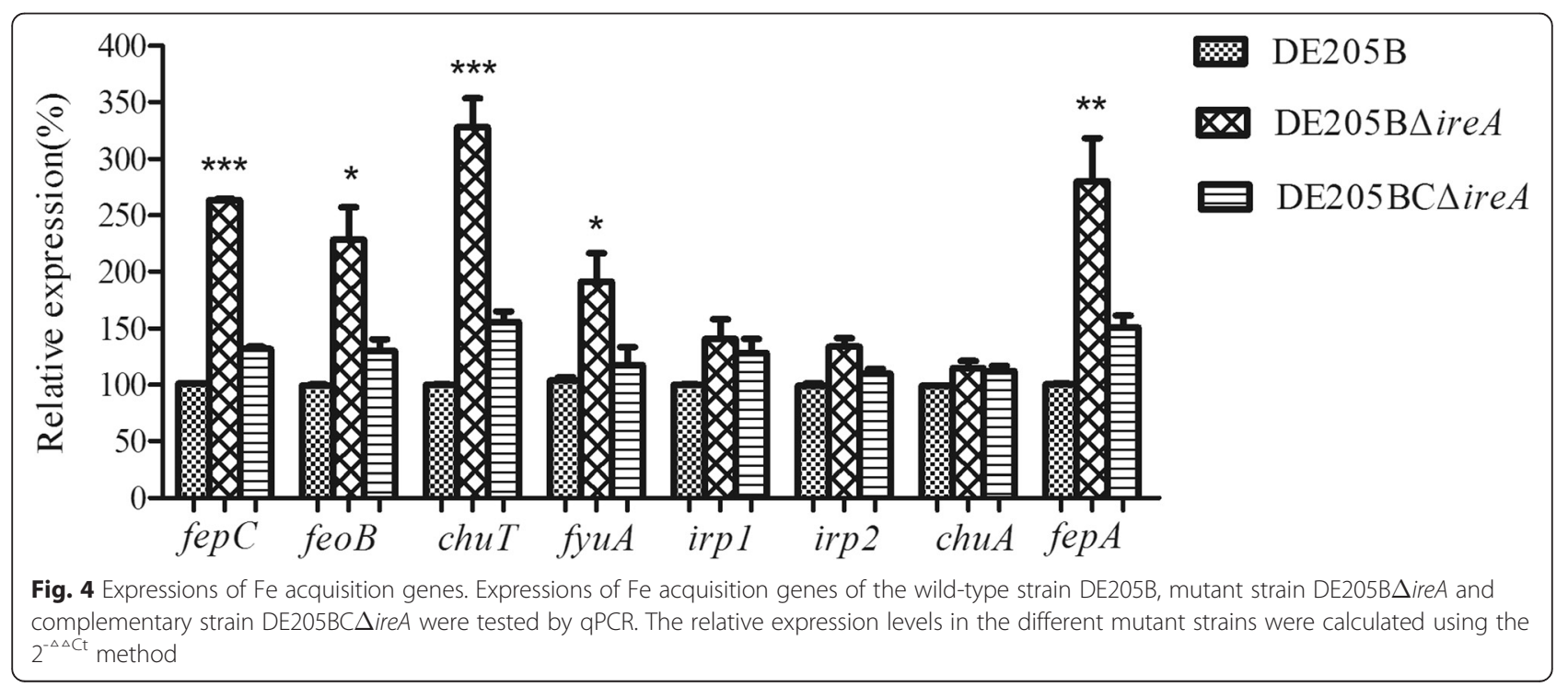




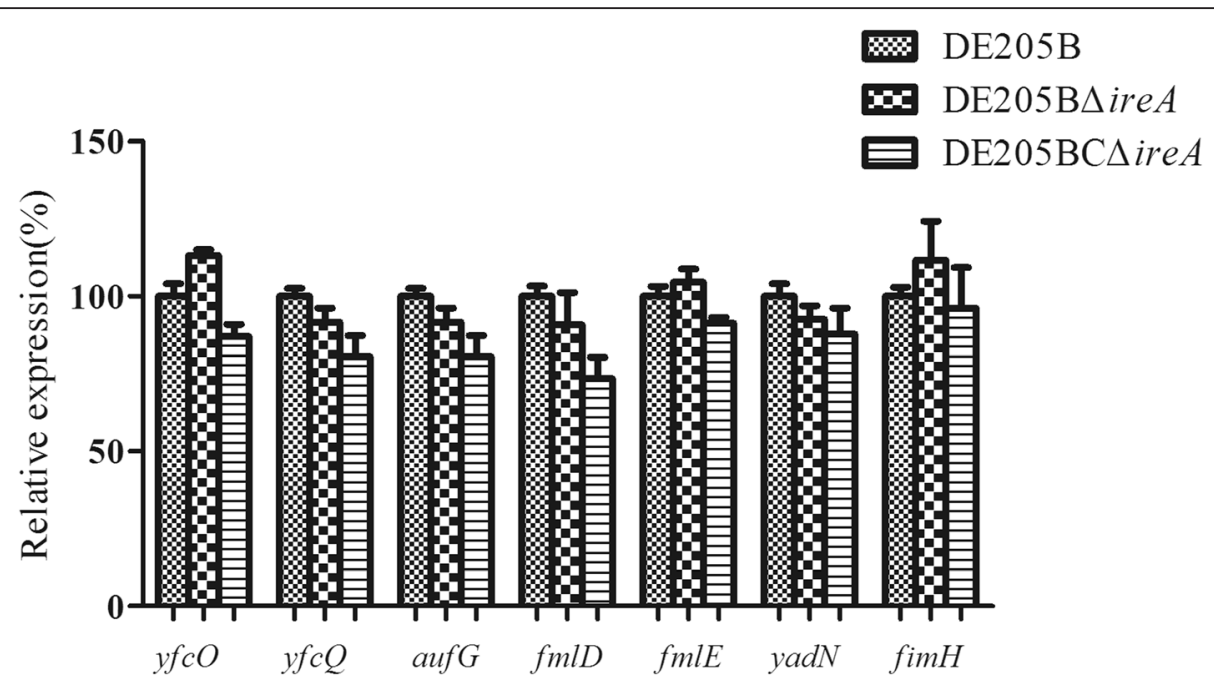

Fig. 5 Expressions of adhesion genes. Expressions of adhesion genes, including $y f c O, y f c Q$, aufG, fmID, fmlE, yadN and fimH, of the wild-type strain DE205B, mutant strain DE205BAireA and complementary strain DE205BCAireA were tested by qPCR. The relative expression levels in the different mutant strains were calculated using the $2^{-\Delta \Delta \mathrm{Ct}}$ method

redundancy of siderophore receptors might reflect their multifunctional roles.

E. coli strains were reported to be classified into four main phylogenetic groups (A, B1, B2, and D) [15]. Virulent ExPEC strains mainly belong to phylogroup B2 and $D$, whereas most commensal strains belong to phylogroup A [16]. The ireA gene was distributed more frequently in the B2 (58.8 \%) and D (55.9\%) groups than in

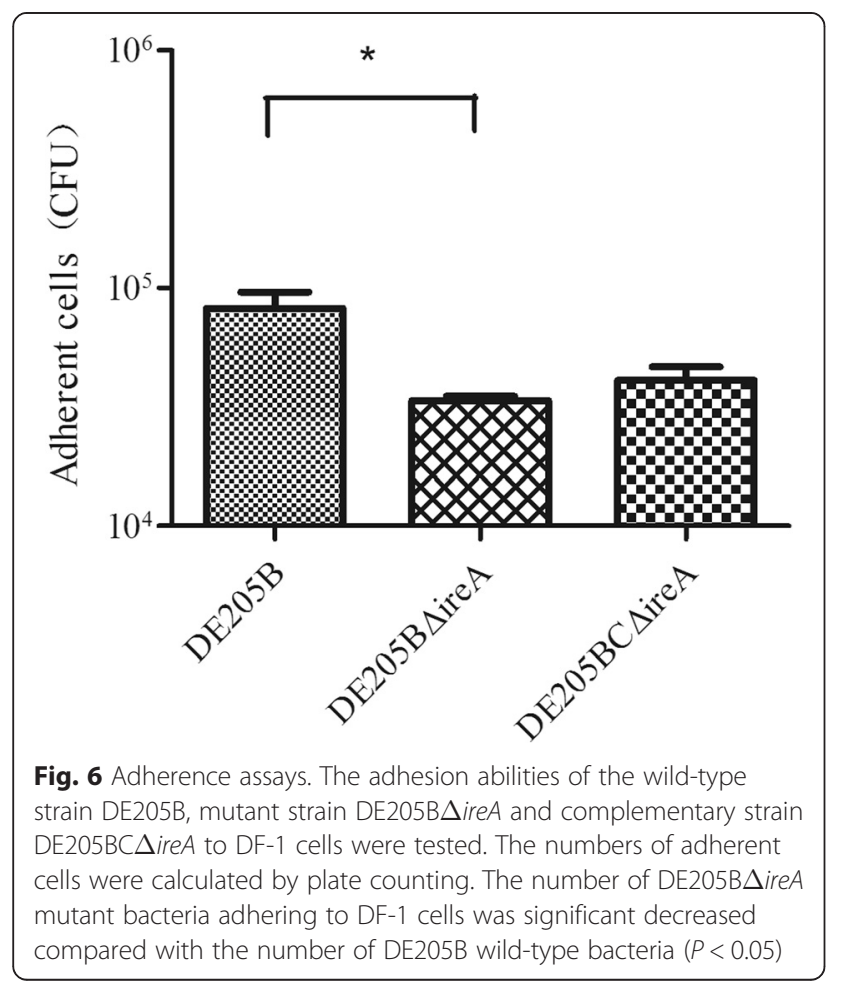

the A (19\%) and B1 (19.2\%) groups, indicating that ireA might be associated with the virulence of the APEC strain DE205B. Thus, the result correlated with that Russo's report: ireA was detected in 13 (26\%) of 50 random clinical isolates from patients and in none $(0 \%)$ of 14 fecal isolates, which presumably represented commensal strains [11]. Taken together, these results indicated that ireA might be a virulence gene in both human and avian ExPEC E. coli strains.

The expression level of ireA in DE205B was decreased in high Fe M9 media compared with that in low $\mathrm{Fe}$ media, indicating that ire $A$ is involved in iron-regulation in this APEC strain. This result agreed with the report of Russo et al., who found that ireA was a iron-regulated virulence gene in the blood- or urine-derived isolates of ExPEC E. coli [11]. Most ferric uptake genes, such as $f e p C$, feoB, chuT, fyuA and fepA were upregulated in the ire $A$ deletion mutant strain. This might represent a compensatory function for ire $A$ gene deletion. Fe acquisition is important for many microorganisms, especially for pathogens that grow in the host, which attempts to limit Fe availability. It is thought that pathogens harbor

Table $2 \mathrm{LD}_{50}$ of wild-type and mutant strains

\begin{tabular}{|c|c|c|c|}
\hline \multirow{2}{*}{$\begin{array}{l}\text { Challenge } \\
\text { dose (CFU/ml) }\end{array}$} & \multicolumn{3}{|c|}{ Duck mortality } \\
\hline & DE205B & DE205B AireA & DE205BC $\Delta$ ireA \\
\hline $10^{8}$ & $7 / 7$ & $7 / 7$ & $7 / 7$ \\
\hline $10^{7}$ & $7 / 7$ & $7 / 7$ & $7 / 7$ \\
\hline $10^{6}$ & $6 / 7$ & $6 / 7$ & $6 / 7$ \\
\hline $10^{5}$ & $3 / 7$ & $2 / 7$ & $1 / 7$ \\
\hline $\mathrm{LD}_{50}$ & $1.74 \times 10^{5}$ & $2.45 \times 10^{5}$ & $3.16 \times 10^{5}$ \\
\hline
\end{tabular}



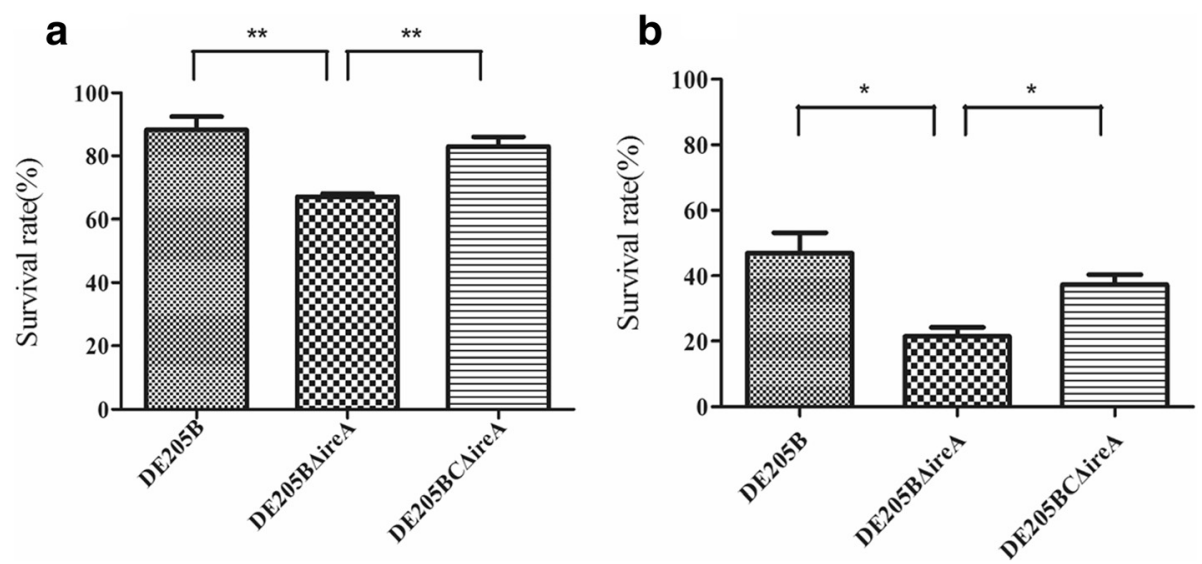

Fig. 7 Resistance to alkaline and high osmolarity. The resistance to alkali (a) and high osmolarity (b) of wild-type strain DE205B, mutant strain DE205B $\Delta$ ireA and complementary strain DE205BC $\Delta$ ireA was tested by exposing the strains to alkaline and high osmolarity conditions and calculating their survival rates. Compared with the wild-type strain, the survival rate of the ireA deletion mutant was reduced $21.17 \%(P<0.01)$ and $25.42(P<0.05)$ under alkaline and high osmolarity conditions, respectively

multiple $\mathrm{Fe}$ acquisition systems to ensure that $\mathrm{Fe}$ is gained from the host cells to provide a selective advantage. Alternatively, certain siderophores, and their cognate receptors, might be more active in certain environments, such as inside or outside the gastrointestinal tract $[17,18]$. Moreover, multiple systems might represent 'alternatives' that protect against the disruption of one system caused by genomic rearrangements or mutations.

In the duck infection experiments, the $\mathrm{LD}_{50}$ showed no significant difference between the wild-type DE205B

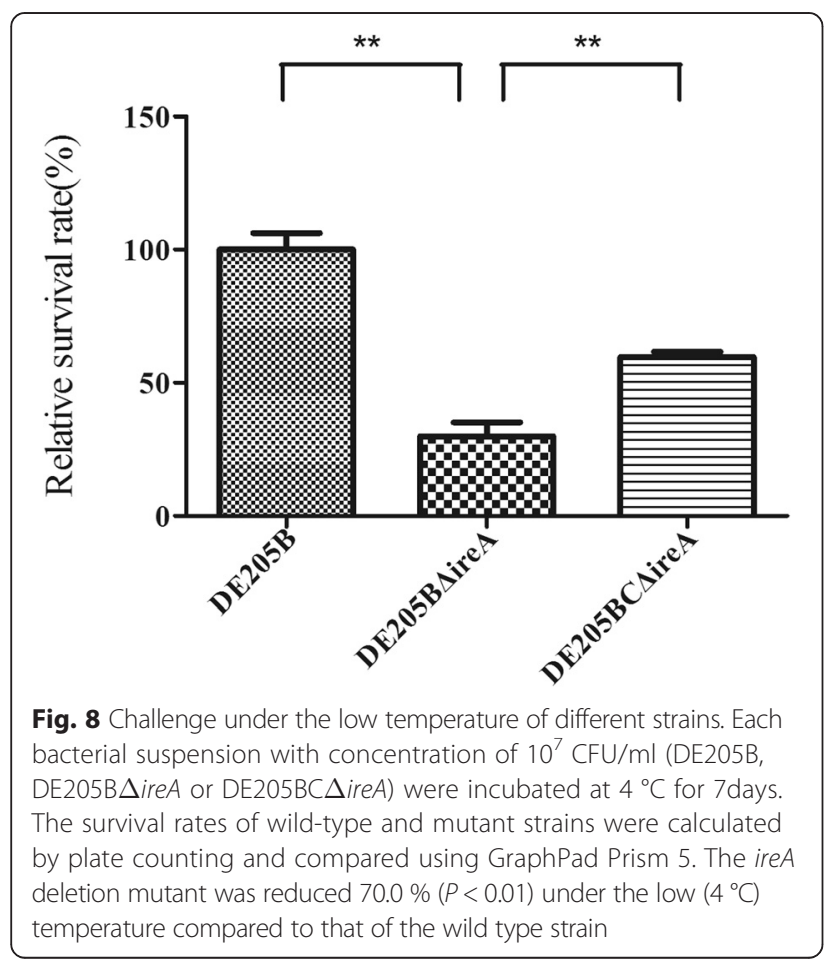

and the ire $A$ deletion mutant strain. Thus, it seemed that ire $A$ deletion had no obvious effect on the virulence of DE205B. However, DE205B has several Fe acquisition systems. We showed that most of the other Fe acquisition genes were upregulated in the ireA gene deletion mutant. Thus, the ireA gene might indeed contribute to the virulence of the APEC strain DE205B, while other Fe acquisition genes displayed compensatory functions when the ireA gene was deleted.

\section{Conclusions}

In summary, the ire $A$ gene was mainly distributed in the more virulent phylogenetic ECOR group B and D. Compared with the wild-type strain, the adhesion and resistance to environmental stress of the ire $A$ deletion mutant were significant decreased. This indicated that ireA is a Fe iron-regulated gene that aids adhesion and stress-resistance in the APEC strain DE205B.

\section{Methods}

Bacterial strains, plasmids and growth conditions

The APEC strain DE205B, which was characterized previously, was isolated from the brain of a duck with neurological symptoms in our previous work [19-22]. Its serotype is $\mathrm{O} 2: \mathrm{K} 1$, which is one of the predominant serotypes of APEC $[9,23]$. All E. coli strains were grown in Luria-Bertani (LB) medium at $37{ }^{\circ} \mathrm{C}$ with shaking or on LB plates with $2 \%$ agar. When necessary, the LB medium was supplemented with appropriate antibiotics: ampicillin (Amp; $100 \mu \mathrm{g} / \mathrm{mL}$ ) and kanamycin (Kan; $50 \mu \mathrm{g} / \mathrm{mL}$ ), unless otherwise specified. Information on the main strains and plasmids used in this study are listed in Table 3. M9 minimal medium was used to assess the expression of ire $A$, which was prepared as 
Table 3 Bacterial strains and plasmids used in the present study

\begin{tabular}{lll}
\hline Strain or plasmid & Characteristics & Reference \\
\hline Strain & & \\
DE205B & O2:K1, NalR & {$[16,19]$} \\
DE205B $\Delta$ ireA & ireA deletion mutant strain & This study \\
DE205BC $\Delta$ ireA & ireA complementary strain & This study \\
Plasmid & & \\
pKD46 & Amp, express $\lambda$ red recombinase & {$[29]$} \\
pKD4 & kan, template plasmid & {$[29]$} \\
pSTV28 & Cm, expression using lac promotor & TAKARA \\
pCP20 & $C m$, Amp, yeast Flp recombinase gene, FLP & {$[29]$} \\
\hline
\end{tabular}

follows: an aliquot was mixed with200 $\mathrm{ml}$ of $5 \times \mathrm{M} 9$ salt solution (containing $\mathrm{Na}_{2} \mathrm{PO}_{4} \cdot 7 \quad \mathrm{H}_{2} \mathrm{O}, 12.8 \mathrm{~g} ; \mathrm{KH}_{2} \mathrm{PO}_{4}$, $3.0 \mathrm{~g}$; NaCl, $0.5 \mathrm{~g} ; \mathrm{NH}_{4} \mathrm{Cl}, 1.0 \mathrm{~g}$ ), $2 \mathrm{ml}$ of $1 \mathrm{M} \mathrm{MgSO}_{4}$, $20 \mathrm{ml}$ of $20 \%$ glucose solution, $0.1 \mathrm{ml}$ of $1 \mathrm{M} \mathrm{CaCl}_{2}$, and then dissolved in $1000 \mathrm{ml}$ double distilled water andfiltrated through a $0.22-\mu \mathrm{m}$ membrane.

\section{Prevalence of ireA among $E$. coli Strains}

The distribution of ireA among 140 E. coli strains (Additional file 1: Table S1) maintained in our laboratory was tested using PCR. The PCR primers were designed using Primer Premier 5. The specific primers ireA-F and ireA-R are listed in Table 4. The PCR conditions were: $5 \mathrm{~min}$ at $95{ }^{\circ} \mathrm{C}$ for initial denaturing; $30 \mathrm{cy}$ cles of $1 \mathrm{~min}$ at $95{ }^{\circ} \mathrm{C}, 30 \mathrm{~s}$ at $50{ }^{\circ} \mathrm{C}$ and $1 \mathrm{~min}$ at $72{ }^{\circ} \mathrm{C}$; followed by $10 \mathrm{~min}$ at $72{ }^{\circ} \mathrm{C}$ for extension. The DNA of DE205B and $d d^{2} \mathrm{H}_{2} \mathrm{O}$ were used as positive and negative templates, respectively. The PCR products were electrophoresed through $1 \%$ agarose gels. Phylogenetic E. coli reference (ECOR) groups of single isolates of the 140 E. coli strains were determined using triplex PCR, as described previously [16, 24].

\section{Expression of the ireA gene}

The expression of the ire $A$ gene was tested by fusion expression and western blotting as previously reported $[25,26]$. The fusion fragment of ire $A$ (including the ire $A$ promoter and $579 \mathrm{bp}$ of ireA sequence) and His tag were inserted into plasmid pET32a(+). The fusion PCR primers P1 and P2 were listed in Table 4. Plasmid pET32a(+) without the fusion fragment was used as a blank control. For immunoblotting, protein samples were subjected to sodium dodecylsulfate polyacrylamide gel electrophoresis (SDS-PAGE) and transferred to polyvinylidene fluoride membranes (Amersham Pharmacia Biotech, Piscataway, NJ, USA), as described previously [27]. Anti-His serum was the primary antibody, horseradish peroxidase-conjugated goat anti-mouse IgG was the secondary antibody and 3,3'-diaminobenzidine was used as the substrate.

\section{Regulation of ireA expression in M9 media}

M9 minimal medium was used to assess the expression of ireA.M9 medium or M9 medium with Fe (0.1 mM $\left.\mathrm{Fe}\left(\mathrm{NO}_{3}\right)_{3}\right)$ were used as low and high Fe content media, respectively. DE205B was cultured in both M9 media to the mid-log phase and the expression of ire $A$ was detected by quantitative real-time reverse transcription PCR(qRT-PCR). The real -time PCR primers were designed by PrimerQuest Tool IDT (http://sg.idtdna.com/ primerquest/Home/Index). Briefly, total RNA was extracted from $1 \mathrm{ml}$ of bacteria culture using the Trizol RNA isolation protocol (Invitrogen, Shanghai, China) and cDNA was amplified by reverse transcription according to the instructions of the primeScript $\mathrm{RT}$ reagent Kit (Takara). Quantitative real-time PCR (qPCR) was carried out using the ABI Prism 7300 and Sequence Detection System software version 1.4 (Applied Biosystems, Foster City, CA, USA), according to the instructions of the SYBR Premix Ex Taq (Takara, Dalian, China). QPCR primers for ireA (QireA-F and QireA-R) are listed in Table 4. DnaE was used as a reference gene. Assays were performed three times. The relative expressions of ire $A$ in different media were calculated using the $2^{-\Delta \Delta \mathrm{Ct}}$ method [28]. Statistical analysis was performed using an unpaired $t$ test in Graphpad Prism 5.0.

\section{Construction of the ireA deletion mutant and complementary strain}

An ireA knockout strain of DE205B was constructed using the lambda red recombinase system described by Datsenko and Wanner [29]. The specific primers ire $A \mathrm{Mu}-\mathrm{F}$ and ire $A \mathrm{Mu}-\mathrm{R}$ were designed to amplify the target gene ireA. The kanamycin resistance gene, which contained sequences homologous to the $5^{\prime}$ and $3^{\prime}$ ends of the target sequence, was amplified using plasmid pKD4 as a template. The PCR products were then transformed by electroporation into DE205B containing the lambda red recombinase expression plasmid pKD46. The transformed bacterial cells were first incubated at $30{ }^{\circ} \mathrm{C}$ for $2 \mathrm{~h}$ in super optimal broth with catabolite repression (SOC) broth, and then grown on LB agar containing kanamycin at $37{ }^{\circ} \mathrm{C}$. Mutants were confirmed by PCR and sequenced using primers $\mathrm{k} 1$ and $\mathrm{k} 2$ (Table 4) in combination with primers ireA-1 and ireA-2 (Table 4) flanking the ire $A$ region. To remove the kanamycin resistance gene, plasmid pCP20 was transformed into the mutant and a kanamycin sensitive mutant strain was selected. Finally, the ireA deletion mutant strain without kanamycin resistance was named as DE205B ireA (Additional file 2: Figure S1).

To construct the complementary strain, the ireA gene, including its putative promoters, was amplified using primers ireACo-F and ireACo-R (Table 4). The following amplification program were used: $5 \mathrm{~min}$ at $95{ }^{\circ} \mathrm{C}$ for 
Table 4 Primers used in the present study

\begin{tabular}{|c|c|c|c|}
\hline Primers & Primer sequence $\left(5^{\prime}-3^{\prime}\right)$ & Gene & Reference \\
\hline ireA-F & AGATACGCTTGTTGTTAC & \multirow[t]{2}{*}{ ireA } & \multirow[t]{2}{*}{ This study } \\
\hline ireA-R & CATTCCATGCTGCTAC & & \\
\hline ireAMu-F & $\begin{array}{l}\text { GTAAATTCCCTCTTTGCTAACGCAAATCATTATC } \\
\text { ATTACGCCTTTGGCAAAGGGAAATTTGTGTAGG } \\
\text { CTGGAGCTGCTTCGA }\end{array}$ & Upstream region of ireA & \multirow[t]{2}{*}{ This study } \\
\hline ireAMu-R & $\begin{array}{l}\text { CATTACTGATTACTACACTGGTACCTGAGGCTC } \\
\text { ACGGCCTCAGGTTGTCTTIATATACTCCATATGA } \\
\text { ATATCCTCCTTAG }\end{array}$ & Downstream region of ireA & \\
\hline ireA-1 & TACTCCCCATCCCCGGGCAA & \multirow[t]{2}{*}{ ireA } & \multirow[t]{2}{*}{ This study } \\
\hline ireA-2 & GGGGGCAGCAATATCCGGTG & & \\
\hline k1 & CAGTCATAGCCGAATAGCCT & \multirow[t]{2}{*}{ kanR } & \multirow[t]{2}{*}[27]{} \\
\hline k2 & CGGTGCCCTGAATGAACTGC & & \\
\hline ireACo-F & TACGGATCCCTGAACAATAGCGAT & \multirow[t]{2}{*}{ ireA } & \multirow[t]{2}{*}{ This study } \\
\hline ireACo-R & GCTAAGCTIITACAACCTGGAAAC & & \\
\hline QireA-F & AAACATGGGATGGCGTACTT & \multirow[t]{2}{*}{ ireA } & \multirow[t]{2}{*}{ This study } \\
\hline QireA-R & AATCAATGGGCCTGACAGATAG & & \\
\hline QFeoB-F & GCACTCTITGTGCATGGTATTC & \multirow[t]{2}{*}{$\mathrm{FeoB}$} & \multirow[t]{2}{*}{ This study } \\
\hline QFeoB-R & TGGCAGCACGGTGTTAAT & & \\
\hline QfepA-F & CAATGCGCCAGAACATAAAGAG & \multirow[t]{2}{*}{ fepA } & \multirow[t]{2}{*}{ This study } \\
\hline QfepA-R & TGTCGAGGTTGCCATACAAG & & \\
\hline QfepC-F & TCGTTACGCCAGCCATTT & \multirow[t]{2}{*}{ fepC } & \multirow[t]{2}{*}{ This study } \\
\hline QfepC-R & TGCAGCGCAGACCATAAA & & \\
\hline QfyuA-F & ATGCCTATGTGGGATGGAATG & \multirow[t]{2}{*}{ fyuA } & \multirow[t]{2}{*}{ This study } \\
\hline QfyuA-R & CCAGTCATCGGTGGTGTATTT & & \\
\hline Qirp1-F & GGCGAACCCTGCTATGTATT & \multirow[t]{2}{*}{ irpl } & \multirow[t]{2}{*}{ This study } \\
\hline Qirp1-R & GTCCATGCAGTACCAGCTAAA & & \\
\hline Qirp2-F & GCGGCTGATTACCAACAATTAC & \multirow[t]{2}{*}{ irp2 } & \multirow[t]{2}{*}{ This study } \\
\hline Qirp2-R & CTGGATCAGGTTGCTCTCTTC & & \\
\hline QChuA-F & TAGGCCACATCAAGGCTAAAC & \multirow[t]{2}{*}{ ChuA } & \multirow[t]{2}{*}{ This study } \\
\hline QChuA-R & CGGCGACAACTATGTCGTATAA & & \\
\hline QdnaE-F & ATGTCGGAGGCGTAAGGCT & $d n a E$ & This study \\
\hline QdnaE-R & TCCAGGGCGTCAGTAAACAA & & \\
\hline P1 & $\begin{array}{l}\text { TTGCCGGAATTCTCAGGGGGCCA } \\
\text { GTCTACTGAATGAG }\end{array}$ & fusion ireA & This study \\
\hline P2 & $\begin{array}{l}\text { ACCGTCGTCGACTTAGTGATGATGATGAT } \\
\text { GATGAGAACCATACAGGGAAGACATAGGG }\end{array}$ & & \\
\hline QyfcO-F & TGCATAACTCGTCATGTCCTGTCTCCGTTG & $y f c O$ & This study \\
\hline QyfcO-R & AGCGTTTCTCAATCGTTCCGCCCGTC & & \\
\hline QyfcQ-F & CCTGCATTTCTACGGCAATTTAC & $y f C Q$ & This study \\
\hline QyfcQ-R & CGGTCACCATCAGGTCTITAC & & \\
\hline QaufG-F & TGCTCTGGCAACATCAGTAG & aufG & This study \\
\hline QaufG-R & ACTAACAGGTAAAGTCAGGAAAGT & & \\
\hline QfmlD-F & CAGGCTCCCTACAGTCATATAAAG & $f m / D$ & This study \\
\hline QfmlD-R & TCGGTGTCTTATCACCAATATCC & & \\
\hline QfmlE-F & CAGTGCCAGACAATTTCCAAC & fmlE & This study \\
\hline QfmlE-R & CCGTTAAATGCAACCTGAACTC & & \\
\hline
\end{tabular}


Table 4 Primers used in the present study (Continued)

\begin{tabular}{lll}
\hline QyadN-F & CCACTGTTAATGGCGGTGTA & yadN \\
QyadN-R & TTAGCCAGGCGAGAAGAAC & This study \\
QfimH-F & CTTATGGCGGCGTGTTATCT & fimH \\
QfimH-R & CGGCTTATCCGTTCTCGAATTA & This study \\
\hline
\end{tabular}

initial denaturing; 35 cycles of $30 \mathrm{~s}$ at $95^{\circ} \mathrm{C}, 30 \mathrm{~s}$ at $55^{\circ}$ $\mathrm{C}$ and $2.5 \mathrm{~min}$ at $72{ }^{\circ} \mathrm{C} ; 10 \mathrm{~min}$ at $72{ }^{\circ} \mathrm{C}$ for extension. The PCR product of ire $A$ gene was purified and subcloned into plasmid pSTV-28. The complementary strain DE205BCDireA was generated by transforming vector pSTV-28-ireA into the deletion mutant (Additional file 3: Figure S2).

\section{Growth curve}

The growths of the DE205B wild-type and mutant strains were compared in LB medium at $37{ }^{\circ} \mathrm{C}$ over a course of $12 \mathrm{~h}$, starting at $10^{7} \mathrm{CFU} / \mathrm{ml}$. Bacterial growth was estimated by plate counting as Colony Forming Units (CFU). Assays were performed three times.

\section{The expression of the Ferric uptake system and adherence genes}

The effect of ireA deletion on the regulation of the ferric uptake system, including $f e p C, f e o B, c h u T, f y u A$, irp1, $\operatorname{irp} 2$, chuA and fepA was detected using qRT- PCR. Seveal adherence genes, including $y f c O, y f c Q$, aufG, fmlD, fmlE, yadN and fimH were also selected to test their expression levels. The wild-type DE205B and the ire A deletion mutant were cultured in LB to mid-log phase and total RNA was extracted from $1 \mathrm{ml}$ of bacterial culture using the Trizol RNA isolation protocol (Gibco BRL, USA, cat. no.15596-026). cDNA was reverse transcribed and real-time PCR was carried out as described above. The qRT-PCR primers for the ferric uptake system and adherence genes are listed in Table 4. The real-time PCR primers were designed unsing PrimerQuest Tool IDT (http://sg.idtdna.com/primerquest/ Home/Index). The relative expression levels of the genes were calculated using the $2^{-\Delta \Delta \mathrm{Ct}}$ method [28]. Assays were performed three times, and the statistical analysis was performed using an unpaired $t$ test in Graphpad Prism 5.0. On the figures, error bars indicate the standard deviation.

\section{Adherence assays}

The adherence assay was performed as described previously [24]. Briefly, chicken embryo fibroblast (CEF) DF-1 cells were seeded at approximately $1 \times 10^{5}$ cells per well in 24-well tissue culture trays (TPP, Shanghai, China) and grown in Dulbecco's modified Eagle medium (DMEM) with $10 \%$ fetal bovine serum at $37{ }^{\circ} \mathrm{C}$ in a $5 \%$ $\mathrm{CO}_{2}$ humidified atmosphere without antibiotics. DF-1 cells were washed once with DMEM and then inoculated with 500 ul of $2 \times 10^{7} \mathrm{CFU} / \mathrm{ml}$ bacteria per well for $2 \mathrm{~h}$ at $37{ }^{\circ} \mathrm{C}$ in the presence of $5 \% \mathrm{CO}_{2}$. The cells were washed, lysed with $\mathrm{dd}_{2} \mathrm{O}$, and the number of bacterial cells was calculated by plate counting. In all assays, wells only containing DF-1 cells were used as negative controls. The adherence assays were conducted three times. The statistical analysis was performed using an unpaired $t$ test in Graphpad Prism 5.0.

\section{Animal infections}

Animal infections were performed as described previously [19, 20]. We purchased 7-day-old ducklings and young duck feeds from Anhui Poultry Farm (Anhui, China). Bacterial strains were cultured to the exponential phase, harvested, washed three times in PBS and then adjusted to the appropriate doses. Twenty-five 7day-old ducks were inoculated intramuscularly $0.2 \mathrm{ml}$ of each bacterial suspension (DE205B, DE205B $\Delta$ ireA or $\mathrm{DE} 205 \mathrm{BC} \triangle$ ire $A)$ at four concentrations $\left(5 \times 10^{8} \mathrm{CFU} /\right.$ $\mathrm{ml}, 5 \times 10^{7} \mathrm{CFU} / \mathrm{ml}, 5 \times 10^{6} \mathrm{CFU} / \mathrm{ml}$, and $5 \times 10^{5} \mathrm{CFU} /$ $\mathrm{ml})$. Assays were performed three times. Seven ducks were used for each dose. Seven ducks were injected with PBS as a negative control. Death of the ducks was monitored for 7 days post infection. We calculated the $\mathrm{LD}_{50}$ of each strain using the method described by Spearman-Karber [30].

\section{Determination of resistance to environmental stress}

Resistance to environmental stress was tested for the wildtype and the mutant strain, as described by La Ragione et al. [31]. Bacteria were cultured in LB broth overnight and harvested by centrifugation. The cells were resuspended in PBS and adjusted to $10^{7} \mathrm{CFU} / \mathrm{ml}$ in PBS. For alkali challenge, $100 \mu \mathrm{l}$ of adjusted cells were mixed with $100 \mu \mathrm{l}$

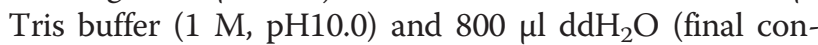
centration, $100 \mathrm{mM}, \mathrm{pH} 10.0)$ and incubated at $37{ }^{\circ} \mathrm{C}$ for $30 \mathrm{~min}$. For high osmolarity endurance, cells were mixed with an equal volume of $4.8 \mathrm{M} \mathrm{NaCl}$ (final concentration, $2.4 \mathrm{M}$ ) and incubated at $37^{\circ} \mathrm{C}$ for $1 \mathrm{~h}$. Bacteria were exposed to PBS (pH 7.0) as a control.

Temperature challenge was performed as previously described with modifications [32], each bacterial suspension, at a concentration of $10^{7} \mathrm{CFU} / \mathrm{ml}$ (DE205B, DE205B $\triangle$ ire A or DE205BC $\triangle$ ireA) was incubated at $4{ }^{\circ} \mathrm{C}$ for 7 days. Assays were performed three times. The survival rates of 
wild-type and mutant strains were calculated by plate counting and compared using GraphPad Prism 5.

\section{Additional files}

Additional file 1: Table S1. Detailed information about the distribution of the ireA gene in APEC strains. (DOCX $21 \mathrm{~kb}$ )

Additional file 2: Figure S1. Technical routes of construction of the ireA deletion mutant. (TIF $98 \mathrm{~kb}$ )

Additional file 3: Figure S2. Technical routes of construction of the ireA complementary strain. (TIF $102 \mathrm{~kb}$ )

\section{Acknowledgements}

Authors are grateful to all of the staff at Key Laboratory Animal Bacteriology of Nanjing Agricultural University for helping conduct the experiments.

\section{Funding}

This work was supported by grants from the National Basic Research Program of China (2015CB554203), the Fund of Priority Academic Program Development of Jiangsu Higher Education Institutions (PAPD), the Fundamental Research Funds for the Central Universities (KYZ201326), the Youth Foundation of the National Natural Science Foundation of China (No.31402213), the Natural Science Foundation of Jiangsu Province, China (No. BK20140686).

\section{Availability of data and materials}

All the data supporting our findings is contained within the manuscript.

\section{Authors' contributions}

$Y L$ carried out the gene deletion and adhesion assays, as well as expression of ireA gene, JD carried out the tests of resistance to environmental stress, $X Z, H W$ and $L H$ carried out the animal infections, JR, LC, DL carried out the QPCR and gene prevelance tests. FT responsible for the growth curve, whole exprements design and draft of the manuscript. All authors read and approved the final manuscript.

\section{Competing interests}

The authors declare that they have no competing interests.

\section{Consent to publish}

Not applicable.

\section{Ethics and consent to participate}

Our experiments were conducted with the permission of the Ministry of Science and Technology of Jiangsu Province. The license number is SYXK(SU) 2010-0005. All efforts were made to minimize animal suffering.

Received: 14 March 2016 Accepted: 10 August 2016

Published online: 17 August 2016

\section{References}

1. Dziva F, Hauser H, Connor TR, van Diemen PM, Prescott G, Langridge GC, Eckert S, Chaudhuri RR, Ewers C, Mellata M, et al. Sequencing and functional annotation of avian pathogenic Escherichia coli serogroup 078 strains reveal the evolution of $\mathrm{E}$. coli lineages pathogenic for poultry via distinct mechanisms. Infect Immun. 2013;81(3):838-49.

2. Dissanayake DR, Octavia S, Lan R. Population structure and virulence content of avian pathogenic Escherichia coli isolated from outbreaks in $\mathrm{Sr}$ Lanka. Vet Microbiol. 2014;168(2-4):403-12.

3. Garenaux A, Houle S, Folch B, Dallaire G, Truesdell M, Lepine F, Doucet N, Dozois CM. Avian lipocalin expression in chickens following Escherichia coli infection and inhibition of avian pathogenic Escherichia coli growth by ExFABP. Vet Immunol Immunopathol. 2013;152(1-2):156-67.

4. Heinemann IU, Jahn M, Jahn D. The biochemistry of heme biosynthesis. Arch Biochem Biophys. 2008:474(2):238-51.

5. Gao Q, Wang X, Xu H, Xu Y, Ling J, Zhang D, Gao S, Liu X. Roles of iron acquisition systems in virulence of extraintestinal pathogenic Escherichia coli: salmochelin and aerobactin contribute more to virulence than heme in a chicken infection model. BMC Microbiol. 2012;12:143.

6. Kouse AB, Righetti F, Kortmann J, Narberhaus F, Murphy ER. RNA-mediated thermoregulation of iron-acquisition genes in Shigella dysenteriae and pathogenic Escherichia coli. PLoS One. 2013;8(5):e63781.

7. Pilarczyk-Zurek M, Chmielarczyk A, Gosiewski T, Tomusiak A, Adamski P, Zwolinska-Wcislo M, Mach T, Heczko PB, Strus M. Possible role of Escherichia coli in propagation and perpetuation of chronic inflammation in ulcerative colitis. BMC Gastroenterol. 2013;13:61.

8. Nagy G, Dobrindt U, Kupfer M, Emody L, Karch H, Hacker J. Expression of hemin receptor molecule ChuA is influenced by $\mathrm{RfaH}$ in uropathogenic Escherichia coli strain 536. Infect Immun. 2001;69(3):1924-8.

9. Li G, Laturnus $C$, Ewers $C$, Wieler LH. Identification of genes required for avian Escherichia coli septicemia by signature-tagged mutagenesis. Infect Immun. 2005;73(5):2818-27.

10. Schouler C, Koffmann F, Amory C, Leroy-Setrin S, Moulin-Schouleur M. Genomic subtraction for the identification of putative new virulence factors of an avian pathogenic Escherichia coli strain of $\mathrm{O} 2$ serogroup. Microbiology. 2004;150(Pt 9):2973-84.

11. Russo TA, Carlino UB, Johnson JR. Identification of a new iron-regulated virulence gene, ireA, in an extraintestinal pathogenic isolate of Escherichia coli. Infect Immun. 2001;69(10):6209-16.

12. Tarr Pl, Bilge SS, Vary Jr JC, Jelacic S, Habeeb RL, Ward TR, Baylor MR, Besser TE. Iha: a novel Escherichia coli 0157:H7 adherence-conferring molecule encoded on a recently acquired chromosomal island of conserved structure. Infect Immun. 2000;68(3):1400-7.

13. Goldberg MB, DiRita VJ, Calderwood SB. Identification of an iron-regulated virulence determinant in Vibrio cholerae, using TnphoA mutagenesis. Infect Immun. 1990;58(1):55-60.

14. Tashima KT, Carroll PA, Rogers MB, Calderwood SB. Relative importance of three iron-regulated outer membrane proteins for in vivo growth of Vibrio cholerae. Infect Immun. 1996;64(5):1756-61.

15. Herzer PJ, Inouye S, Inouye M, Whittam TS. Phylogenetic distribution of branched RNA-linked multicopy single-stranded DNA among natural isolates of Escherichia coli. J Bacteriol. 1990;172(11):6175-81.

16. Clermont $\mathrm{O}$, Bonacorsi $\mathrm{S}$, Bingen E. Rapid and simple determination of the Escherichia coli phylogenetic group. Appl Environ Microbiol. 2000;66(10): 4555-8.

17. Bearden SW, Perry RD. The Yfe system of Yersinia pestis transports iron and manganese and is required for full virulence of plaque. Mol Microbiol. 1999; 32(2):403-14.

18. Tsolis RM, Baumler AJ, Heffron F, Stojiljkovic I. Contribution of TonB- and Feo-mediated iron uptake to growth of Salmonella typhimurium in the mouse. Infect Immun. 1996;64(11):4549-56.

19. Wang S, Niu C, Shi Z, Xia Y, Yaqoob M, Dai J, Lu C. Effects of ibeA deletion on virulence and biofilm formation of avian pathogenic Escherichia coli. Infect Immun. 2011;79(1):279-87.

20. Wang S, Xia Y, Dai J, Shi Z, Kou Y, Li H, Bao Y, Lu C. Novel roles for autotransporter adhesin AatA of avian pathogenic Escherichia coli: colonization during infection and cell aggregation. FEMS Immunol Med Microbiol. 2011:63(3):328-38.

21. Zhuge X, Wang S, Fan H, Pan Z, Ren J, Yi L, Meng Q, Yang X, Lu C, Dai J. Characterization and functional analysis of AatB, a novel autotransporter adhesin and virulence factor of avian pathogenic Escherichia coli. Infect Immun. 2013:81(7):2437-47.

22. Wang S, Shi Z, Xia Y, Li H, Kou Y, Bao Y, Dai J, Lu C. IbeB is involved in the invasion and pathogenicity of avian pathogenic Escherichia coli. Vet Microbiol. 2012;159(3-4):411-9.

23. Blanco JE, Blanco M, Mora A, Jansen WH, Garcia V, Vazquez ML, Blanco J. Serotypes of Escherichia coli isolated from septicaemic chickens in Galicia (northwest Spain). Vet Microbiol. 1998;61(3):229-35.

24. Dai J, Wang S, Guerlebeck D, Laturnus C, Guenther S, Shi Z, Lu C, Ewers C. Suppression subtractive hybridization identifies an autotransporter adhesin gene of E. coli IMT5155 specifically associated with avian pathogenic Escherichia coli (APEC). BMC Microbiol. 2010;10:236.

25. Urban JH, Vogel J. Translational control and target recognition by Escherichia coli small RNAs in vivo. Nucleic Acids Res. 2007;35(3):1018-37.

26. Wu Z, Wu C, Shao J, Zhu Z, Wang W, Zhang W, Tang M, Pei N, Fan H, Li $J$, et al. The Streptococcus suis transcriptional landscape reveals adaptation mechanisms in pig blood and cerebrospinal fluid. RNA. 2014; 20(6):882-98 
27. Wang S, Bao Y, Meng Q, Xia Y, Zhao Y, Wang Y, Tang F, ZhuGe X, Yu S, Han $X$, et al. IbeR facilitates stress-resistance, invasion and pathogenicity of avian pathogenic Escherichia coli. PLoS One. 2015;10(3):e0119698.

28. Ali-Benali MA, Alary R, Joudrier P, Gautier MF. Comparative expression of five Lea Genes during wheat seed development and in response to abiotic stresses by real-time quantitative RT-PCR. Biochim Biophys Acta. 2005; 1730(1):56-65.

29. Datsenko KA, Wanner BL. One-step inactivation of chromosomal genes in Escherichia coli K-12 using PCR products. Proc Natl Acad Sci U S A. 2000; 97(12):6640-5.

30. Fung KP, Wong TW. Estimation of quantal dose response of drugs by the Spearman-Karber method: a computer program written in BASIC. J Singapore Paediatr Soc. 1989;31(3-4):129-32.

31. La Ragione RM, Sayers AR, Woodward MJ. The role of fimbriae and flagella in the colonization, invasion and persistence of Escherichia coli 078:K80 in the day-old-chick model. Epidemiol Infect. 2000;124(3):351-63.

32. McLeod A, Mage I, Heir E, Axelsson L, Holck AL. Effect of relevant environmental stresses on survival of enterohemorrhagic Escherichia coli in dry-fermented sausage. Int J Food Microbiol. 2016;229:15-23.

\section{Submit your next manuscript to BioMed Central} and we will help you at every step:

- We accept pre-submission inquiries

- Our selector tool helps you to find the most relevant journal

- We provide round the clock customer support

- Convenient online submission

- Thorough peer review

- Inclusion in PubMed and all major indexing services

- Maximum visibility for your research

Submit your manuscript at www.biomedcentral.com/submit 